\title{
Calcium intake is associated with decreased prevalence of periodontal disease in young Japanese women
}

\author{
Keiko Tanaka ${ }^{*}$, Yoshihiro Miyake ${ }^{2}$, Hitomi Okubo ${ }^{3}$, Takashi Hanioka ${ }^{4}$, Satoshi Sasaki ${ }^{5}$, Nobuyuki Miyatake ${ }^{1}$
} and Masashi Arakawa ${ }^{6}$

\begin{abstract}
Background: We investigated the relationships between calcium intake and the prevalence of periodontal disease. Methods: This cross-sectional study included 1162 women with a mean age of 31.5 years. Information on dietary factors was collected using a diet history questionnaire during pregnancy. Oral examinations were performed between one and twelve months postpartum. Periodontal disease was defined as positive if a woman had at least one tooth with a pocket depth of $4.0 \mathrm{~mm}$ or deeper. Adjustment was made for age, region of residence, smoking status, toothbrushing frequency, use of an interdental brush, household income, and education.
\end{abstract}

Results: Compared with the lowest quartile of calcium intake, the highest quartile was significantly associated with a lower prevalence of periodontal disease; however, the inverse linear trend fell just short of the significance level: the adjusted odds ratio was 0.53 (95\% confidence interval: $0.30-0.94, P$ for trend $=0.07$ ).

Conclusions: Our findings suggest that higher calcium intake may be inversely associated with the prevalence of periodontal disease.

Keywords: Calcium, Cross-sectional studies, Periodontal disease, Women

\section{Background}

Periodontal disease is a chronic condition characterized by loss of tooth-supporting connective tissue and alveolar bone [1]. A complex relationship among bacterial, host, behavioral and environmental factors determines the development and progress of the disease [2]. As with various other chronic diseases, intake of foods and nutrients is considered an important factor, but epidemiological evidence on the relationship between diet and periodontal disease has been limited, and the results have been inconsistent [3,4].

Calcium is the most abundant mineral in the human body. Although the majority of calcium in the body is in the structure of bones and teeth, the remaining calcium performs functions so essential to life that they take first priority over bone mineralization [5]. Calcium status in

\footnotetext{
* Correspondence: keikotanaka@med.kagawa-u.ac.jp

'Department of Hygiene, Faculty of Medicine, Kagawa University, Miki,

Kagawa 761-0793, Japan

Full list of author information is available at the end of the article
}

humans is likely to influence bone health, including alveolar bone health. Calcium is one of the relatively more widely studied nutrients in relation to periodontal disease [6-12]. A US cross-sectional study using data from the Third National Health and Nutrition Examination Survey observed a significant positive association between low dietary calcium intake and periodontal disease among young males and females (20 to 30 years of age) and older males (40 to 59 years of age) [8]. In older Danish adults, a higher intake of calcium from dairy products, but not from foods other than dairy products, was significantly inversely associated with the prevalence of periodontal disease [10]. In a longitudinal study among the elderly in Japan, an inverse dose-response relationship between serum calcium levels and periodontal disease was observed in smokers but not in non-smokers [11]. On the other hand, no association was observed between dietary calcium intake and periodontal disease in a case-control study of German adults [12]. Thus, the results on the association between calcium intake and periodontal disease 
have been inconsistent. Further evidence is needed to clarify the influence of calcium intake on periodontal disease.

In the present study, we assessed the relationship between calcium intake and the prevalence of periodontal disease among young Japanese women, using the data set of the Kyushu Okinawa Maternal and Child Health Study (KOMCHS).

\section{Methods \\ Study population}

The KOMCHS is an ongoing prospective prebirth cohort study that investigates risk and preventive factors for maternal and child health problems such as oral health and allergic disorders. Eligible subjects were those women who became pregnant in one of seven prefectures on Kyushu Island in southern Japan or Okinawa Prefecture between April 2007 and March 2008. At 423 obstetric hospitals, a set of leaflets explaining the $\mathrm{KOMCHS}$, an application form to participate in the study, and a self-addressed and stamped return envelope were distributed to pregnant women, insofar as this was possible. Pregnant women who intended to participate in the KOMCHS returned the application form to the data management center. In the end, a total of 1757 pregnant women between the 5th and 39th week of pregnancy gave their written informed consent to participate and also completed the baseline survey. A flowchart depicting the study population included in our analysis is provided in Figure 1. The ethics committee of the Faculty of Medicine, Fukuoka University approved the KOMCHS. The STROBE (Strengthening the Reporting of

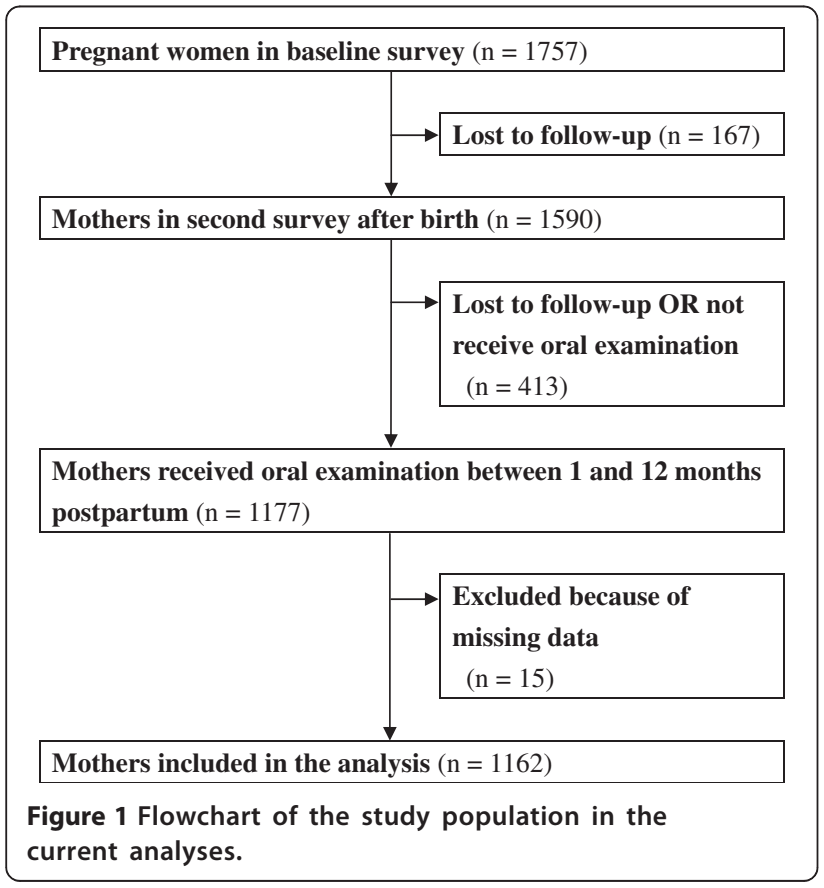

Observational studies in Epidemiology) guidelines were followed.

\section{Outcome variable}

Oral examinations to evaluate the condition of periodontal tissue between one and twelve months postpartum were performed by dental hygienists. Examination was performed under good natural light using a dental mirror and a Community Periodontal Index (CPI) probe (YDM Corp., Tokyo, Japan). Probing pocket depth (PPD) was determined with a CPI probe at six sites per tooth for six teeth: the right first molar, right first incisor, and left first molar in the maxilla and the right first molar, left first incisor, and left first molar in the mandible. When the target tooth was missing, the second molar in the same side or the first incisor in the opposite side was examined. The deepest PPD was recorded for each tooth. Periodontal disease was defined as positive if a woman had at least one tooth with a PPD of $4.0 \mathrm{~mm}$ or deeper.

\section{Exposure variables and covariates}

In the baseline survey, each participant filled out a twopart questionnaire and mailed it to the data management center; all data were derived from a single questionnaire mailed directly to the participants. Research technicians completed missing or unclear data by telephone interview. The first part of the questionnaire elicited information on age, region of residence, smoking habit status, toothbrushing frequency, use of an interdental brush, household income, and educational level. The second part of the questionnaire was a semi-quantitative, comprehensive diet history questionnaire (DHQ) that assessed dietary habits during the preceding month $[13,14]$.

Estimates of daily intake of foods (total of 150 foods), energy, and selected nutrients were calculated using an ad hoc computer algorithm for the DHQ based on the Standard Tables of Food Composition in Japan [15]. Information on dietary supplements was not used due to the lack of a reliable composition table for dietary supplements in Japan. Also, only a small number of participants $(6.1 \%)$ used calcium supplements at least once per week. According to a validation study of 92 females aged 31 to 69 years, Pearson's correlation coefficient between the DHQ and 16-day weighted dietary records was 0.56 for calcium [16]. Energy-adjusted intake by the residual method was used for the analyses [17].

\section{Statistical analysis}

Intake of calcium was categorized into quartiles on the basis of the distribution in 1162 subjects. Age, region of residence, smoking status, toothbrushing frequency, use of an interdental brush, household income, and educational level were selected $a$ priori as potential confounding factors. Region of residence was classified into three 
categories (Fukuoka Prefecture, other than Fukuoka Prefecture on Kyushu Island, and Okinawa Prefecture), smoking status into two (never and ever), toothbrushing frequency into three $(<2,2$, and $\geq 3$ times/day), use of an interdental brush into two (no and yes), household income into three $(<4,000,000,4,000,000-5,999,999$, and $\geq 6,000,000$ yen/year), educational level into three $(<13,13-14$, and $\geq 15$ years $)$. Age was used as a continuous variable.

Logistic regression analysis was performed to estimate crude odds ratios (ORs) and their confidence intervals (CIs) for periodontal disease in relation to calcium intake. Multiple logistic regression analysis was employed to adjust for potential confounding factors. Trend of association was assessed by a logistic regression model in which the median value in each quartile of calcium intake was assigned as the representative score. Two-sided $P$ values less than 0.05 were considered statistically significant. All statistical analyses were performed using the SAS software package version 9.3 (SAS Institute, Inc., Cary, NC, USA). The statistical power calculation was performed using QUANTO version 1.2 [18].

\section{Results}

The prevalence of periodontal disease among the 1162 women was $11.4 \%$. The mean age of the participants was 31.5 years (Table 1 ). About $70 \%$ of women were never smokers. Toothbrushing two and three or more times per day was reported for $50.4 \%$ and $36.8 \%$ of participants, respectively. An interdental brush was used by about $46 \%$ of women. Mean daily total energy intake was $7396.0 \mathrm{~kJ}$, and mean energy-adjusted intake of calcium was $500.2 \mathrm{mg}$.

The crude OR for periodontal disease in relation to every one-year increase in age was 1.04 (95\% CI: 1.00-1.09) (Table 2). Compared with living in Fukuoka Prefecture, living in a prefecture in Kyushu other than Fukuoka Prefecture was associated with increased prevalence of periodontal disease. Compared with the lowest quartile of calcium intake, the highest quartile was significantly associated with a lower prevalence of periodontal disease. After adjustment for confounding factors under study, the inverse association was more evident: the adjusted OR between the extreme quartiles was 0.53 (95\% CI: 0.30-0.94). The inverse linear trend between calcium intake and periodontal disease, however, fell just short of the significance level ( $P$ for linear trend $=0.07$ ).

Statistical power calculation revealed that, using our sample size, we could detect an association between calcium intake and periodontal disease for an OR of 0.518 with an accuracy of more than $80 \%$.

No significant differences between never and ever smokers were observed in the association of calcium intake with the prevalence of periodontal disease $(P=0.22$,
Table 1 Distribution of characteristics of 1162 women, KOMCHS, Japan

\begin{tabular}{|c|c|}
\hline Variable & Number $(\%)$ or mean \pm SD \\
\hline Age (years) & $31.5 \pm 4.2$ \\
\hline \multicolumn{2}{|l|}{ Region of residence } \\
\hline Fukuoka Prefecture & $734(63.2)$ \\
\hline Other than Fukuoka Prefecture in Kyushu & $321(27.6)$ \\
\hline Okinawa Prefecture & $107(9.2)$ \\
\hline \multicolumn{2}{|l|}{ Smoking status } \\
\hline Never & $818(70.4)$ \\
\hline Ever & $344(29.6)$ \\
\hline \multicolumn{2}{|l|}{ Toothbrushing frequency (times/day) } \\
\hline$<2$ & $149(12.8)$ \\
\hline 2 & $586(50.4)$ \\
\hline$\geq 3$ & $427(36.8)$ \\
\hline \multicolumn{2}{|l|}{ Use of an interdental brush } \\
\hline No & $627(54.0)$ \\
\hline Yes & $535(46.0)$ \\
\hline \multicolumn{2}{|l|}{ Household income (yen/year) } \\
\hline$<4,000,000$ & $374(32.2)$ \\
\hline $4,000,000-5,999,999$ & $418(36.0)$ \\
\hline$\geq 6,000,000$ & $370(31.8)$ \\
\hline \multicolumn{2}{|l|}{ Educational level (years) } \\
\hline$<13$ & $239(20.6)$ \\
\hline $13-14$ & $386(33.2)$ \\
\hline$\geq 15$ & $537(46.2)$ \\
\hline Total energy intake (kJ/day) & $7396.0 \pm 1955.5$ \\
\hline Calcium intake $(\mathrm{mg} / \text { day })^{*}$ & $500.2 \pm 220.9$ \\
\hline
\end{tabular}

*Calcium intake was adjusted for total energy intake using the residual method.

0.40 , and 0.98 for homogeneity of OR in the second, third, and highest quartiles, respectively).

\section{Discussion}

In this study, we found that a higher intake of calcium was independently associated with a lower prevalence of periodontal disease among young Japanese women. Our results were in partial agreement with those from the Third National Health and Nutrition Examination Survey, which showed a significant inverse dose-response relationship between calcium intake as assessed by means of a 24-hour dietary recall and periodontal disease [8], but our results were inconsistent with those of a German study that showed no association between calcium intake as assessed by means of a seven-day food record and periodontal disease [12].

A US cross-sectional study reported that an inverse correlation between dietary calcium intake as assessed by means of a 24-hour dietary recall and periodontal 
Table 2 Odds ratios and 95\% confidence intervals for periodontal disease 1162 women, KOMCHS, Japan

\begin{tabular}{|c|c|c|c|c|c|}
\hline & Prevalence & Crude OR $(95 \% \mathrm{Cl})$ & $P$ value & Adjusted OR $(95 \% \mathrm{Cl})^{\dagger}$ & $P$ value \\
\hline Age (years) & & $1.04(1.00,1.09)$ & 0.06 & $1.04(0.99,1.09)$ & 0.09 \\
\hline \multicolumn{6}{|l|}{ Region of residence } \\
\hline Fukuoka Prefecture & $49 / 734(6.7 \%)$ & 1.00 & & 1.00 & \\
\hline Other than Fukuoka Prefecture in Kyushu & $80 / 321(24.9 \%)$ & $4.64(3.17,6.85)$ & $<0.0001$ & $4.84(3.28,7.24)$ & $<0.0001$ \\
\hline Okinawa Prefecture & $3 / 107(2.8 \%)$ & $0.40(0.10,1.13)$ & 0.13 & $0.41(0.10,1.14)$ & 0.14 \\
\hline \multicolumn{6}{|l|}{ Smoking status } \\
\hline Never & $88 / 818(10.8 \%)$ & 1.00 & & 1.00 & \\
\hline Ever & $44 / 344(12.8 \%)$ & $1.22(0.82,1.78)$ & 0.32 & $1.51(0.99,2.29)$ & 0.05 \\
\hline \multicolumn{6}{|l|}{ Toothbrushing frequency (times/day) } \\
\hline$<2$ & $17 / 149(11.4 \%)$ & 1.00 & & 1.00 & \\
\hline 2 & $58 / 586(9.9 \%)$ & $0.85(0.49,1.55)$ & 0.59 & $0.84(0.46,1.57)$ & 0.56 \\
\hline$\geq 3$ & $57 / 427(13.4 \%)$ & $1.20(0.69,2.19)$ & 0.54 & $1.05(0.57,2.01)$ & 0.87 \\
\hline \multicolumn{6}{|l|}{ Use of an interdental brush } \\
\hline No & $66 / 627(10.5 \%)$ & 1.00 & & 1.00 & \\
\hline Yes & $66 / 535(12.3 \%)$ & $1.20(0.83,1.72)$ & 0.33 & $1.14(0.77,1.69)$ & 0.51 \\
\hline \multicolumn{6}{|l|}{ Household income (yen/year) } \\
\hline$<4,000,000$ & $42 / 374(11.2 \%)$ & 1.00 & & 1.00 & \\
\hline $4,000,000-5,999,999$ & $42 / 418(10.1 \%)$ & $0.88(0.56,1.39)$ & 0.59 & $0.88(0.54,1.43)$ & 0.61 \\
\hline$\geq 6,000,000$ & $48 / 370(13.0 \%)$ & $1.18(0.76,1.84)$ & 0.47 & $1.13(0.69,1.87)$ & 0.63 \\
\hline \multicolumn{6}{|l|}{ Educational level (years) } \\
\hline$<13$ & $24 / 239(10.0 \%)$ & 1.00 & & 1.00 & \\
\hline $13-14$ & 49/386 (12.7\%) & $1.30(0.78,2.22)$ & 0.32 & $1.34(0.78,2.38)$ & 0.30 \\
\hline$\geq 15$ & $59 / 537(11.0 \%)$ & $1.11(0.68,1.86)$ & 0.69 & $1.17(0.68,2.06)$ & 0.58 \\
\hline \multicolumn{6}{|l|}{ Calcium intake $(\mathrm{mg} / \text { day })^{*}$} \\
\hline 1st quartile $(338.2,-230.7-393.4)$ & $39 / 290(13.4 \%)$ & 1.00 & & 1.00 & \\
\hline 2nd quartile $(436.3,393.5-479.8)$ & 29/291 (10.0\%) & $0.71(0.42,1.18)$ & 0.19 & $0.66(0.38,1.13)$ & 0.13 \\
\hline 3rd quartile (521.0, 479.9-584.4) & $40 / 290(13.8 \%)$ & $1.03(0.64,1.66)$ & 0.90 & $0.92(0.55,1.52)$ & 0.73 \\
\hline 4th quartile $(667.7,584.5-1478.5)$ & 24/291 (8.2\%) & $0.58(0.33,0.98)$ & 0.05 & $0.53(0.30,0.94)$ & 0.03 \\
\hline$P$ for trend & & 0.11 & & 0.07 & \\
\hline
\end{tabular}

${ }^{*}$ Quartile medians and range adjusted for energy intake by the residual method are given in parentheses.

${ }^{\dagger}$ Adjusted for age, region of residence, smoking status, toothbrushing frequency, use of interdental brush, household income, and educational level.

index as assessed based on visual and radiographic data was of borderline significance $(r=-0.24, P<0.075)$, while serum calcium concentration was not significantly correlated with periodontal index $(r=0.16)$ [6]. These findings are in partial agreement with our results. Nevertheless, it should be noted that the above-mentioned studies used different definitions of outcome, study populations, exposure assessment methods, and confounding factors, thus limiting the feasibility of inter-study comparisons. In particular, the difference in average calcium intake between Japanese and Western populations should be taken into account when interpreting our results. According to the National Health and Nutrition Survey in Japan, the average daily per capita intake of calcium was $512 \mathrm{mg}$ [19]. In contrast, for US women in 1999-2004, the average daily per capita intake of calcium was $756 \mathrm{mg}$ [20]. The results of the present study suggest that, even at the relatively low levels habitually consumed in the Japanese population, calcium might have beneficial effects on periodontal disease.

Periodontal disease is characterized by the loss of toothsupporting structures. In particular, the loss of alveolar bone is one of the most important hallmarks of periodontal disease. Calcium intake influences bone mineral density [21]. A cross-sectional study among young Japanese women showed a positive association between calcium intake and bone mineral density [22]. Increased intake of calcium might prevent bone loss because calcium suppresses the secretion of parathyroid hormone which leads to bone resorption [21]. 
Our study had certain methodological strengths. Study subjects were homogeneous in gender and age group. We were also able to control for relevant confounding factors. It is possible, however, that our results remain confounded by other potentially important factors, such as patterns of dental visits and alcohol consumption.

Several limitations should also be considered. The current study design was cross-sectional, and therefore the temporal nature of the association between calcium intake and periodontal disease could not be examined. We could not estimate the participation rate because the exact number of eligible pregnant women who were provided with a set of leaflets explaining the KOMCHS, an application form, and a self-addressed and stamped return envelope by the 423 collaborating obstetric hospitals is not available. We were also not able to assess the differences between participants and non-participants because information on personal characteristics such as age and socioeconomic status among non-participants is not available. Our subjects were probably not a representative sample of Japanese women in the general population, however. In fact, educational levels in the current study population were higher than in the general population. According to the 2000 population census of Japan, the proportions of women aged 30 to 34 years in Fukuoka Prefecture with $<13,13-14, \geq 15$, and an unknown number of years of education were $52.0 \%$, 31.5\%, $11.8 \%$, and $4.8 \%$, respectively [23]. The corresponding figures for the current study were $20.6 \%, 33.2 \%, 46.2 \%$, and $0.0 \%$, respectively. In addition, the prevalence of periodontal disease in our study population (11.4\%) appeared to be lower than that in the sample of women aged 30 to 34 years in the National Survey of Dental Diseases, conducted in 2011 (14.3\%), in which periodontal disease was likewise defined as one or more periodontal sites with a PPD of $4.0 \mathrm{~mm}$ or deeper [24]. With regard to dietary intake, however, calcium intake in this study population (500 $\mathrm{mg} /$ day) is similar to that in the general population (512 mg/day) [19].

Our DHQ could only approximate consumption and was designed to assess dietary intake for one month prior to completing the questionnaire. We believe that the possibility of non-differential exposure misclassification would introduce a bias toward the null. In the present study, assessment of diet was performed during pregnancy. Substantial changes in diet in the previous month were experienced by 341 pregnant women because of nausea gravidarum (322 women), maternal and fetal health (18 women), and other reasons ( 1 woman). The results of a sensitivity analysis which excluded these 341 women were similar to those in the overall analysis: the adjusted OR between extreme quartiles was 0.51 (95\% CI: 0.26-0.99, $P$ for trend $=0.14)$. In the present study, data on serum calcium concentrations were not available.
In the current study, oral examinations were performed by dental hygienists. The dental hygienists were given detailed criteria for performing the examinations, but they received no specific training aimed at standardizing the procedures. In addition, no reliability assessment of measurements was carried out in the present study. Therefore, it is unknown whether intra- and interexaminer consistency was established. Further, because partial mouth recording was used in the present study, the prevalence of periodontal disease may have been underestimated. Moreover, our case definition of periodontal disease was based solely on the measurement of PPD, that is, the distance from the gingival margin to the base of the gingival sulcus or periodontal pocket. Measurements of PPD and clinical attachment level correlate well in many groups, especially younger populations, and both are accepted as measures of periodontal status [25].

\section{Conclusions}

Findings from this cross-sectional study among young adult Japanese women suggest that calcium intake may be associated with periodontal disease. Calcium intake might have beneficial effects on periodontal disease even at the relatively low levels of intake that are typical in Japan. Further studies are required to confirm these findings and to understand the mechanisms behind the observed association between calcium intake and periodontal disease.

\section{Abbreviations}

Cl: Confidence interval; CPI: Community periodontal index; DHQ: Diet history questionnaire; KOMCHS: Kyushu Okinawa Maternal and Child Health Study; OR: Odds ratio; PPD: Probing pocket depth.

\section{Competing interests}

The authors declare that they have no competing interests.

\section{Authors' contributions}

$K T, Y M$, and MA contributed to the study concept and design and the data acquisition. $\mathrm{HO}$ and SS were responsible for the estimation of dietary factors. TH contributed to the dental survey. KT was responsible for the analysis and interpretation of data and the drafting of the manuscript. NM assisted in manuscript preparation. All authors read and approved the final manuscript.

\section{Acknowledgements}

The authors would like to acknowledge the Kyushu Branch of the Japan Allergy Foundation, the Fukuoka Association of Obstetricians \&

Gynecologists, the Okinawa Association of Obstetricians \& Gynecologists, the Miyazaki Association of Obstetricians \& Gynecologists, the Oita Association of Obstetricians \& Gynecologists, the Kumamoto Association of Obstetricians \& Gynecologists, the Nagasaki Association of Obstetricians \& Gynecologists, the Kagoshima Association of Obstetricians \& Gynecologists, the Saga Association of Obstetricians \& Gynecologists, the Fukuoka Society of Obstetrics and Gynecology, the Okinawa Society of Obstetrics and Gynecology, the Fukuoka Dental Hygienists' Association, the Okinawa Dental Hygienists' Association, the Miyazaki Dental Hygienists' Association, the Oita Dental Hygienists' Association, the Kumamoto Dental Hygienists' Association, the Nagasaki Dental Hygienists' Association, the Kagoshima Dental Hygienists' Association, the Saga Dental Hygienists' Association, the Fukuoka City Government, and the Fukuoka City Medical Association for their valuable support. 
This study was supported by JSPS KAKENHI Grant Numbers 19590606, 20791654, 21590673, 22592355, 22119507, 24390158, 25463275, and 25670305; by Health and Labour Sciences Research Grants for Research on Allergic Disease and Immunology from the Ministry of Health, Labour, and Welfare, Japan; by Meiji Co. Ltd.; by the Food Science Institute Foundation; and by Society for Women's Science Research (13-B2-005).

\section{Author details}

'Department of Hygiene, Faculty of Medicine, Kagawa University, Miki, Kagawa 761-0793, Japan. ${ }^{2}$ Department of Public Health, Ehime University Graduate School of Medicine, Ehime, Japan. ${ }^{3}$ Department of Health Promotion, National Institute of Public Health, Saitama, Japan. ${ }^{4}$ Department of Preventive and Public Health Dentistry, Fukuoka Dental College, Fukuoka, Japan. ${ }^{5}$ Department of Social and Preventive Epidemiology, School of Public Health, The University of Tokyo, Tokyo, Japan. ${ }^{6}$ Course of Wellness, Graduate School of Tourism Sciences, University of the Ryukyus, Okinawa, Japan.

Received: 24 July 2014 Accepted: 12 November 2014

Published: 24 November 2014

\section{References}

1. Pihlstrom BL, Michalowicz BS, Johnson NW: Periodontal diseases. Lancet 2005, 366:1809-1820.

2. Nunn ME: Understanding the etiology of periodontitis: an overview of periodontal risk factors. Periodontol 2000 2003, 32:11-23.

3. Al-Zahrani MS, Bissada NF, Borawski EA: Diet and periodontitis. J Int Acad Periodontol 2005, 7:21-26.

4. Neiva RF, Steigenga J, Al-Shammari KF, Wang HL: Effects of specific nutrients on periodontal disease onset, progression and treatment. J Clin Periodontol 2003, 30:579-589.

5. Palmer CA: Diet and Nutrition in Oral Health. NJ: Prentice Hall: Upper Saddle River; 2003:87-90.

6. Freeland JH, Cousins RJ, Schwartz R: Relationship of mineral status and intake to periodontal disease. Am J Clin Nutr 1976, 29:745-749.

7. Uhrbom E, Jacobson L: Calcium and periodontitis: clinical effect of calcium medication. J Clin Periodontol 1984, 11:230-241.

8. Nishida M, Grossi SG, Dunford RG, Ho AW, Trevisan M, Genco RJ: Calcium and the risk for periodontal disease. J Periodontol 2000, 71:1057-1066.

9. Miley DD, Garcia MN, Hildebolt CF, Shannon WD, Couture RA, Anderson Spearie CL, Dixon DA, Langenwalter EM, Mueller C, Civitelli R: Crosssectional study of vitamin $\mathrm{D}$ and calcium supplementation effects on chronic periodontitis. J Periodontol 2009, 80:1433-1439.

10. Adegboye AR, Christensen LB, Holm-Pedersen P, Avlund K, Boucher BJ, Heitmann BL: Intake of dairy products in relation to periodontitis in older Danish adults. Nutrients 2012, 4:1219-1229.

11. Yoshihara A, Iwasaki M, Miyazaki H: Mineral content of calcium and magnesium in the serum and longitudinal periodontal progression in Japanese elderly smokers. J Clin Periodontol 2011, 38:992-997.

12. Staudte H, Kranz S, Völpel A, Schütze J, Sigusch BW: Comparison of nutrient intake between patients with periodontitis and healthy subjects. Quintessence Int 2012, 43:907-916.

13. Sasaki S, Yanagibori R, Amano K: Self-administered diet history questionnaire developed for health education: a relative validation of the test-version by comparison with 3-day diet record in women. J Epidemiol 1998, 8:203-215.

14. Sasaki S, Ushio F, Amano K, Morihara M, Todoriki O, Uehara Y, Toyooka E: Serum biomarker-based validation of a self-administered diet history questionnaire for Japanese subjects. J Nutr Sci Vitaminol (Tokyo) 2000, 46:285-296.

15. Science and Technology Agency: Standard Tables of Food Composition in Japan, 5th Revised and enlarged edn. Tokyo, Japan: Printing Bureau of the Ministry of Finance; 2005. in Japanese.

16. Kobayashi S, Honda S, Murakami K, Sasaki S, Okubo H, Hirota N, Notsu A, Fukui M, Date C: Both comprehensive and brief self-administered diet history questionnaires satisfactorily rank nutrient intakes in Japanese adults. J Epidemiol 2012, 22:151-159.

17. Willett W, Stampfer MJ: Total energy intake: implications for epidemiologic analyses. Am J Epidemiol 1986, 124:17-27.

18. Gauderman WJ: Sample size requirements for matched case-control studies of gene-environment interaction. Stat Med 2002, 21:35-50.
19. The Study Circle for Health and Nutrition Information: The National Health and Nutrition Survey in Japan, 2009. Tokyo: Daiichi Shuppan; 2012:60.

20. Wang Y, Li S: Worldwide trends in dairy production and consumption and calcium intake: is promoting consumption of dairy products a sustainable solution for inadequate calcium intake? Food Nutr Bull 2008, 29:172-185.

21. Zhu K, Prince RL: Calcium and bone. Clin Biochem 2012, 45:936-942.

22. Ito $\mathrm{S}$, Ishida $\mathrm{H}$, Uenishi $\mathrm{K}$, Murakami K, Sasaki S: The relationship between habitual dietary phosphorus and calcium intake, and bone mineral density in young Japanese women: a cross-sectional study. Asia Pac J Clin Nutr 2011, 20:411-417.

23. Statistics Bureau: Ministry of Public Management, Home Affairs, Post and Telecommunications, Japan: 2000 Population Census of Japan, Vol. 3-2-27, Labour Force Status of Population, Industry (Major Groups) of Employed Persons, and Education. Osaka-fu. Japan Statistical Association: Tokyo; 2002.

24. Japanese Society for Dental Health: Report on the Survey of Dental Diseases (2011). Tokyo: Japanese Society for Oral Health; 2013.

25. Page RC, Eke PI: Case definitions for use in population-based surveillance of periodontitis. J Periodontol 2007, 78(7 Suppl):1387-1399.

doi:10.1186/1475-2891-13-109

Cite this article as: Tanaka et al:: Calcium intake is associated with decreased prevalence of periodontal disease in young Japanese women. Nutrition Journal 2014 13:109.

\section{Submit your next manuscript to BioMed Central and take full advantage of:}

- Convenient online submission

- Thorough peer review

- No space constraints or color figure charges

- Immediate publication on acceptance

- Inclusion in PubMed, CAS, Scopus and Google Scholar

- Research which is freely available for redistribution

Submit your manuscript at www.biomedcentral.com/submit
C Biomed Central 